\title{
Dietary Relationships among Feral Horses, Cattle, and Pronghorn in Southeastern Oregon
}

\author{
MICHAEL L. MCINNIS AND MARTIN VAVRA
}

\begin{abstract}
Management of sympatric ungulates on multiple use lands requires knowledge of how species exploit resources available to them. We examined seasonal food habits, dietary overlap, and dietary quality of sympatric feral horses (Equus caballus), cattle (Bos taurus), and pronghorn (Antilocapra americana) in southeastern Oregon from May 1979 through March 1981. Seasonal diets of each ungulate species were determined by microhistological analysis of feces. At least $88 \%$ of the mean annual diets of horses and cattle consisted of grasses. Principal species consumed by these ungulates were bottlebrush squirreltail (Situnion hystrix (Nutt.) J.G. Smith), bearded bluebunch wheatgrass (A gropyron spicatum (Pursh) Scribn. \& Smith), and Thurber needlegrass (Stipa thurberiana Piper). Because dietary overlap between horses and cattle was high each season (62-78\%), we concluded a strong potential existed for exploitative competition under conditions of limited forage availability. Pronghorn diets consisted largely of woody sagebrush (Artemisia) species in fall and winter, and a mixture of forbs in spring and summer. Dietary overlap between horses and pronghorn varied from $7 \%$ (summer) to $26 \%$ (winter). Overlap between cattle and pronghorn varied from $8 \%$ (winter) to $25 \%$ (spring). These lower levels of overlap indicate a wider buffer between noncompetitive coexistence and exploitative competition. Pronghorn generally selected diets containing higher levels of crude protein (CP) and lower levels of acid-detergent fiber (ADF) than horses or cattle. We observed few differences in seasonal dietary quality between horses and cattle.
\end{abstract}

Key Words: food habits, diet quality, fecal analysis, ungulates, sympatry, resource partitioning

The principal goal of resource management on multiple use lands is to provide sustained yields of a variety of plant and animal products. The Wild Free-Roaming Horse and Burro Act (PL 92-195) of 1971 recognized feral horses as "an integral part of the natural system", and mandated their preservation and protection "in thriving ecological balance with other legitimate uses of public lands." Prior to 1971, unrestricted removal of feral horses from public rangelands tended to maintain populations at relatively low levels. After passage of PL 92-195, McGuire (1973) predicted the law would reverse this tendency. Indeed, annual rates of increase for 2 herds in southeastern Oregon are estimated to be about 20\% (Eberhardt et al. 1982). At this rate, a herd will more than double in 4 years. Such population growth may increase the potential of competition for resources shared by horses, cattle, and pronghorn.

To achieve an ecological balance among communities of sympatric organisms, management must be based on knowledge of how species use and partition resources available to them (Hudson 1976). Dietary partitioning among ungulates may be explained on the basis of (1) body size, (2) type of digestive system (cecal or

\footnotetext{
Authors are assistant professor, Dept. Rangeland Resources, Oregon State Univ, Corvallis 97331; and superintendent, Eastern Oregon Agricultural Research Center, Star Rt. 1, 4.5 Hwy. 205, Burns 97720.

This research was a cooperation effort jointly funded by the Vale, Oregon District of the Bureau of Land Management (Contract No. YA-512-CT8-137), and the Eastern Oregon Agricultural Research Center. Published as Oregon Agricultural Experiment Station Technical Paper No. 7784.

The authors gratefully acknowledge Jerry $A$. Wilcox (Wild Horse Specialist, Vale

BLM) for his support of the study; and Ronney C. Slater for technical assistance.

Manuscript accepted 4 June 1986.
}

ruminant), (3) rumino-reticular volume, and (4) mouth size (Hanley 1982, Demment and Van Soest 1985). This food selection framework predicts large cecal digestors (horses) and small ruminants (pronghorn) be positioned at opposite ends of dietary gradient with graminoids at one end and forbs and shrubs at the other. Based on this reasoning cattle should be positioned nearer to horses than pronghorn (Hanley and Hanley 1982). In view of this framework, we examined seasonal food habits, dietary overlap, forage quality, and dietary quality of sympatric horses, cattle, and pronghorn in southeastern Oregon. We hypothesized (1) horses and cattle would select highly similar diets consisting principally of graminoids, while pronghorn would select diets composed largely of forbs and shrubs, and (2) pronghorn would select diets of higher nutritional quality than horses or cattle.

\section{Study Area}

The study was conducted approximately $130 \mathrm{~km}$ southeast of Burns, Ore., on a $670-\mathrm{km}^{2}$ area administered by the Vale District, Bureau of Land Management. Elevations vary from $1,200 \mathrm{~m}$ at Burns Junction to 1,700 $\mathrm{m}$ on nearby Sheepshead Mountains.

Mean annual precipitation at Burns Junction is $208 \mathrm{~mm}$. Summer thunderstorms contribute to total precipitation but most rain falls during spring. Snow accumulates throughout winter and is greatest during January. Annual snowfall at Burns Junction averages $107 \mathrm{~mm}$. Mean annual temperature is $9.6^{\circ} \mathrm{C}$, with mean monthly extremes of $-2.5^{\circ} \mathrm{C}$ (January) and $22.5^{\circ} \mathrm{C}$ (July).

The study area lies within the salt desert shrub and shrub-steppe regions described by Franklin and Dyrness (1973). Salt desert shrub communities are restricted to low elevations and are often intermingled with communities dominated by Wyoming big sagebrush (Artemisia tridentata spp. wyomingensis Beetle.) Principal shrubs of these salt desert shrub communities are shadscale saltbrush (Atriplex confertifolia (Torr. \& Frem) Wats.), spiny hopsage (Grayia spinosa (Hook). Moq.), and bud sagebrush (Artemisa spinescens Eat.). Important grasses associated with these shrubs are bottlebrush squirreltail and cheatgrass brome (Bromus tectorum L.).

Uplands support shrub-steppe communities dominated by Wyoming big sagebrush. Principal grasses are bearded bluebunch wheatgrass, Sandberg bluegrass (Poa sandbergii Vassey), Thurber needlegrass, and bottlebrush squirreltail. Low sagebrush (Artemisia arbuscula Nutt.)/Sandberg bluegrass communities are common on the shallow, stony soils of ridgetops.

\section{Methods}

Field work extended from May 1979 through March 1981. Monthly data were pooled seasonally as follows: spring, March-May; summer, June-August; fall, September-November; and winter, December-February.

\section{Food Habits}

Botanical composition of diets was determined by microscopic examination of fresh fecal material (Sparks and Malechek 1968). Five independent fecal samples per month were prepared for each studied species of ungulate. Each sample was a composite (equal by weight) of feces collected from 6-12 observed individuals so that 30-60 individuals of each species were sampled monthly. One 
Table 1. Mean percent compodition ( \pm S.E.) of important ${ }^{1}$ forages in seasonal diets of foral horees.

\begin{tabular}{|c|c|c|c|c|c|c|c|}
\hline Forage class/species & $\begin{array}{c}\text { Summer } 1979 \\
(93)^{2}\end{array}$ & $\begin{array}{l}\text { Fall } 1979 \\
(102)\end{array}$ & $\begin{array}{l}\text { Winter } 1980 \\
\text { (122) }\end{array}$ & $\begin{array}{l}\text { Spring } 1980 \\
\text { (136) }\end{array}$ & $\begin{array}{l}\text { Summer } 1980 \\
(114)\end{array}$ & $\begin{array}{c}\text { Fall } 1980 \\
(139)\end{array}$ & $\begin{array}{l}\text { Winter } 1981 \\
\text { (141) }\end{array}$ \\
\hline $\begin{array}{l}\text { Graminoids } \\
\text { Agropyron spicatum } \\
\text { Bromus tectorum } \\
\text { Poa sandbergii } \\
\text { Sitanion hystrix } \\
\text { Stipa thurberiana } \\
\text { Other graminoids } \\
\text { Unknown graminoids } \\
\text { Total graminoids }\end{array}$ & $\begin{array}{r}32(8.3) \\
7(4.3) \\
3(1.1) \\
29(7.5) \\
14(1.9) \\
2(0.3) \\
4(0.4) \\
91(1.0)\end{array}$ & $\begin{array}{c}14(0.9) \\
3(1.2) \\
9(2.5) \\
43(3.7) \\
17(3.8) \\
T^{3} \\
3(0.6) \\
89(2.4)\end{array}$ & $\begin{array}{r}9(0.7) \\
2(1.6) \\
13(8.0) \\
45(8.2) \\
15(1.2) \\
2(0.5) \\
4(0.1) \\
90(1.9)\end{array}$ & $\begin{array}{r}24(4.9) \\
15(5.8) \\
11(3.2) \\
24(2.4) \\
19(0.4) \\
2(1.2) \\
3(0.4) \\
98(0.3)\end{array}$ & $\begin{array}{r}24(1.7) \\
18(4.2) \\
1(0.7) \\
8(2.1) \\
28(4.5) \\
4(2.7) \\
4(0.8) \\
87(3.5)\end{array}$ & $\begin{array}{c}6(0.6) \\
4(2.0) \\
15(3.2) \\
36(1.1) \\
13(5.7) \\
0 \\
5(0.8) \\
79(2.6)\end{array}$ & $\begin{array}{c}9(3.0) \\
3(0.3) \\
11(6.3) \\
49(5.4) \\
7(0.6) \\
T \\
7(0.4) \\
86(3.2)\end{array}$ \\
\hline $\begin{array}{l}\text { Forbs } \\
\text { Phlox hoodii } \\
\text { Phlox longifolia } \\
\text { Unknown forbs } \\
\text { Total forbs }\end{array}$ & $\begin{array}{l}4(1.1) \\
1(0.3) \\
2(0.1) \\
7(1.1)\end{array}$ & $\begin{array}{l}5(2.4) \\
1(0.2) \\
1(0.5) \\
7(2.7)\end{array}$ & $\begin{array}{l}5(1.9) \\
1(0.7) \\
1(0.3) \\
7(1.4)\end{array}$ & $\begin{array}{l}1(0.5) \\
T \\
1(0.2) \\
2(0.6)\end{array}$ & $\begin{array}{l}5(1.1) \\
T \\
1(0.6) \\
6(0.9)\end{array}$ & $\begin{array}{c}13(1.8) \\
0 \\
1(0.7) \\
14(2.1)\end{array}$ & $\begin{array}{r}10(2.1) \\
1(0.9) \\
1(0.6) \\
12(2.5)\end{array}$ \\
\hline $\begin{array}{l}\text { Shrubs } \\
\text { Artemisia arbuscula/ } A \\
\text { tridentata ssp. } \\
\text { wyomingensis } \\
\text { Atriplex confertifolia } \\
\text { Ceratoides lanata } \\
\text { Total shrubs }\end{array}$ & $\begin{array}{l}0 \\
T \\
2(0.9) \\
2(0.9)\end{array}$ & $\begin{array}{l}1(0.5) \\
T \\
3(2.3) \\
4(1.9)\end{array}$ & $\begin{array}{l}T \\
3(1.3) \\
T \\
3(1.9)\end{array}$ & $\begin{array}{l}0 \\
T \\
T \\
T\end{array}$ & $\begin{array}{l}0 \\
1(0.8) \\
6(2.1) \\
7(2.7)\end{array}$ & $\begin{array}{l}T \\
4(0.6) \\
3(1.4) \\
7(1.0)\end{array}$ & $\begin{array}{l}0 \\
1(0.6) \\
1(0.9) \\
2(1.3)\end{array}$ \\
\hline
\end{tabular}

'Species comprising at least $1 \%$ of any seasonal diet; complete species list found in Mclnnis (1985).

2Number of fecal groups comprising the mean of 3 samples per season.

${ }^{3}$ Trace; less than $0.5 \%$.

microscope slide was prepared for each sample and examined at the rate of 20 systematically selected fields at $100 x$ magnification. Identification of plant species was based on comparison with epidermal characteristics of reference materials. Monthly means were expressed as percent relative density and pooled to yield 3 independent replications per season. We judged fecal analysis the only practical method for determining diets of these free-ranging herbivores. Although forage species may vary in digestibility and discernibility, we believed fecal analysis provided adequate data to rank forages important to the studied herbivores (McInnis et al. 1983). Highly digestible and poorly discernible species may be underestimated.

\section{Dietary Overlap}

Percent overlap of a forage species shared by a pair of ungulate species (horses vs. cattle; horses vs. pronghorn; and cattle vs. pronghorn) was defined as the lesser percentage consumed by 1 ungulate. Total dietary overlap was calculated within each season as the sum of overlaps of individual forages (Whittaker 1975, Anthony and Smith 1977, Hurlbert 1978). This index may vary from $0 \%$ (no overlap) to $100 \%$ (complete overlap).

\section{Forage Quality}

Samples of important forage species were collected concomitant with fecal material. Between 1-4 samples (50-100 g each) of plant parts selected by studied ungulates were analyzed each season for CP (Kjeldahl nitrogen $\times 6.25)$ and ADF (Van Soest and Wine 1967). Crude protein is correlated with desirable nutritional components, and serves as a reliable index of a plant's nutritional value (Sullivan 1962). Acid-detergent fiber represents the fibrous portion of a feed and shows a reasonably strong inverse relationship with digestibility and dietary quality (Van Soest 1963). Data were expressed on a percent dry matter basis and averaged by species within seasons over years.

\section{Dietary Quality}

Percent $\mathrm{CP}$ and ADF in seasonal diets of each ungulate species was calculated following Westoby (1974):

$$
N_{i}=\Sigma_{a_{i j} \mathbf{z}_{j}}
$$

where $\mathrm{Ni}$ is the dietary composition of the ith nutrient, $\mathrm{a}_{\mathrm{ij}}$ is the ith nutrient content of the jth forage species and $\mathrm{xj}$ is the dry weight composition of the $\mathrm{jth}$ forage species. $\mathrm{N}_{\mathrm{i}}$ was calculated for each mean monthly diet yielding 3 values per season for each ungulate species. Occasionally ungulates consumed plants not analyzed for nutrient content. When this occurred for a species in which previous and subsequent seasonal values of $a_{\mathrm{ij}}$ were established, the mean value was used. For species not analyzed, values of similar species were used. Mean values of a forage class were used for unidentified species. In this manner, $100 \%$ of each diet was accounted for.

Data were analyzed as a completely randomized design using factorial analysis of variance to examine effects of the 3 ungulate species and 7 seasons on dietary CP and ADF. Differences among means were established $(P<0.05)$ with Duncan's multiple range test (Steel and Torrie 1960).

\section{Food Habits}

\section{Results}

Feral horses were predominantly grazers throughout the year, and grasses comprised $88 \%$ of pooled seasonal diets. We observed little variation in total grass consumption among most seasons, but usage peaked in spring (Table 1). The principal grass consumed was bottlebrush squirreltail, forming a mean annual composition of $33 \%$. Following in order of importance on an annual basis were bearded bluebunch wheatgrass (17\%), Thurber needlegrass (16\%), Sandberg bluegrass (9\%), and cheatgrass brome (7\%). Usage of these species varied seasonally. Bearded bluebunch wheatgrass and cheatgrass brome together comprised roughly $40 \%$ of combined spring and summer diets, but were used lightly during fall and winter. Consumption of bottlebrush squirreltail followed an opposite pattern and accounted for nearly $50 \%$ of pooled winter diets.

Cattle primarily consumed grass throughout the year. Grasses, forbs, and shrubs formed 89,4 , and $7 \%$, respectively, of the mean annual diet (Table 2). When pooled across seasons and years, bearded bluebunch wheatgrass and bottlebrush squirreltail respectively formed 20 and $40 \%$ of the diet. On an annual basis, these species were followed in order of importance by cheatgrass brome (9\%), and Sandberg bluegrass (5\%). Consumption of bearded bluebunch wheatgrass, cheatgrass brome, and Sandberg bluegrass was higher in pooled spring-summer diets than fall or winter. Conver- 
Table 2. Mean pereent composition ( \pm S.E.) of important' forages in seasonal diets of cattle.

\begin{tabular}{|c|c|c|c|c|c|c|c|}
\hline Forage class/species & $\begin{array}{c}\text { Summer } 1979 \\
(101)^{2}\end{array}$ & $\begin{array}{l}\text { Fall } 1979 \\
\text { (116) }\end{array}$ & $\begin{array}{l}\text { Winter } 1980 \\
(94)\end{array}$ & $\begin{array}{l}\text { Spring } 1980 \\
(99)\end{array}$ & $\begin{array}{l}\text { Summer } 1980 \\
\text { (117) }\end{array}$ & $\begin{array}{l}\text { Fall } 1980 \\
(156)\end{array}$ & $\begin{array}{l}\text { Winter } 1981 \\
\text { (126) }\end{array}$ \\
\hline $\begin{array}{l}\text { Graminoids } \\
\text { Agropyron spicatum } \\
\text { Bromus tectorum } \\
\text { Poa sandbergii } \\
\text { Sitanion hystrix } \\
\text { Stipa thurberiana } \\
\text { Other graminoids } \\
\text { Unknown graminoids } \\
\text { Total graminoids }\end{array}$ & $\begin{array}{r}40(8.6) \\
7(2.3) \\
7(2.5) \\
16(3.6) \\
4(0.9) \\
12(6.0) \\
5(1.2) \\
91(1.9)\end{array}$ & $\begin{array}{r}10(1.8) \\
6(1.3) \\
4(1.0) \\
58(3.3) \\
11(3.1) \\
0 \\
3(0.5) \\
92(1.1)\end{array}$ & $\begin{array}{r}7(2.7) \\
8(0.8) \\
1(0.5) \\
61(3.8) \\
3(0.8) \\
1(0.6) \\
3(0.8) \\
84(4.8)\end{array}$ & $\begin{array}{r}19(4.6) \\
16(4.1) \\
8(2.6) \\
31(6.5) \\
4(0.9) \\
6(3.0) \\
7(2.4) \\
91(1.6)\end{array}$ & $\begin{array}{r}36(2.1) \\
18(1.8) \\
11(2.7) \\
9(2.9) \\
7(1.6) \\
5(0.8) \\
8(4.3) \\
94(1.7)\end{array}$ & $\begin{array}{r}15(2.0) \\
7(2.8) \\
3(1.7) \\
50(3.7) \\
1(0.3) \\
1(0.9) \\
7(0.7) \\
84(0.9)\end{array}$ & $\begin{array}{r}15(2.3) \\
2(1.2) \\
4(0.5) \\
58(2.6) \\
1(0.8) \\
1(0.4) \\
6(0.5) \\
87(0.8)\end{array}$ \\
\hline $\begin{array}{l}\text { Forbs } \\
\text { Phlox hoodii } \\
\text { Phlox longifolia } \\
\text { Other forbs } \\
\text { Unknown forbs } \\
\text { Total forbs }\end{array}$ & $\begin{array}{l}2(1.3) \\
1(0.6) \\
0 \\
4(0.9) \\
7(1.2)\end{array}$ & $\begin{array}{l}1(0.5) \\
0 \\
1(0.8) \\
1(0.6) \\
3(0.8)\end{array}$ & $\begin{array}{l}\mathrm{T}^{3} \\
0 \\
0 \\
1(0.2) \\
1(0.3)\end{array}$ & $\begin{array}{l}2(0.4) \\
1(0.6) \\
3(0.8) \\
3(0.9) \\
9(1.9)\end{array}$ & $\begin{array}{l}2(0.9) \\
1(0.5) \\
0 \\
3(0.9) \\
6(1.8)\end{array}$ & $\begin{array}{l}0 \\
0 \\
0 \\
3(1.3) \\
3(1.3)\end{array}$ & $\begin{array}{l}0 \\
0 \\
0 \\
1(0.7) \\
1(0.7)\end{array}$ \\
\hline $\begin{array}{l}\text { Shrubs } \\
\text { Artemisia arbuscula/A } \\
\text { tridentata spp. } \\
\text { wyomingensis } \\
\text { Atriplex confertifolia } \\
\text { Ceratoides lanata } \\
\text { Total shrubs }\end{array}$ & $\begin{array}{l}\mathrm{T} \\
2(0.4) \\
\mathrm{T} \\
2(0.9)\end{array}$ & $\begin{array}{l}T \\
3(0.6) \\
2(1.1) \\
5(1.3)\end{array}$ & $\begin{array}{c}0 \\
14(4.1) \\
1(0.5) \\
15(4.6)\end{array}$ & $\begin{array}{l}\mathrm{T} \\
0 \\
\mathbf{T} \\
\mathrm{T}\end{array}$ & $\begin{array}{l}\mathbf{T} \\
\mathbf{0} \\
\mathbf{T} \\
\mathbf{T}\end{array}$ & $\begin{array}{c}0 \\
11(1.9) \\
2(0.1) \\
13(2.0)\end{array}$ & $\begin{array}{l}0 \\
12(0.2) \\
0 \\
12(0.2)\end{array}$ \\
\hline
\end{tabular}

ISpecies comprising at least $1 \%$ of any seasonal diet; complete species list found in McInnis (1985).

${ }^{2}$ Number of fecal groups comprising the mean of 3 samples per season.

${ }^{3}$ Trace; less than $0.5 \%$.

sely, consumption of bottlebrush squirreltail was greater during the fall-winter period. Use of forbs and shrubs varied seasonally. Forbs occurred more frequently in spring and summer diets than fall and winter, while shrub consumption generally followed the reverse trend. Shadscale saltbrush was the most heavily consumed shrub, and formed $13 \%$ of pooled winter diets.
Unlike horses and cattle, pronghorn consumed mainly forbs and shrubs throughout the year. Mean annual dietary composition of grasses, forbs, and shrubs was $13 \%, 44 \%$, and $42 \%$, respectively. Seasonal use of these forage classes fluctuated widely, and coincided with the availability of forbs. Forb use peaked during summer months, then declined through fall and winter (Table 3 ).

Table 3. Mean percent composition ( \pm S.E.) of important ${ }^{1}$ forages in seasonal diets of pronghorn.

\begin{tabular}{|c|c|c|c|c|c|c|c|}
\hline Forage class/species & $\begin{array}{c}\text { Summer } 1979 \\
(84)^{2}\end{array}$ & $\begin{array}{l}\text { Fall } 1979 \\
(132)\end{array}$ & $\begin{array}{l}\text { Winter } 1980 \\
(80)\end{array}$ & $\begin{array}{l}\text { Spring } 1980 \\
(52)\end{array}$ & $\begin{array}{l}\text { Summer } 1980 \\
(127)\end{array}$ & $\begin{array}{c}\text { Fall } 1980 \\
(149)\end{array}$ & $\begin{array}{l}\text { Winter } 1981 \\
\text { (147) }\end{array}$ \\
\hline $\begin{array}{l}\text { Graminoids } \\
\text { Bromus tectorum } \\
\text { Poa sandbergii } \\
\text { Sitanion hystrix } \\
\text { Other graminoids } \\
\text { Unknown graminoids } \\
\text { Total graminoids }\end{array}$ & $\begin{array}{l}1(0.2) \\
2(0.8) \\
1(0.5) \\
T \\
0 \\
4(0.4)\end{array}$ & $\begin{array}{l}7(0.9) \\
5(0.8) \\
1(0.6) \\
T \\
T \\
13(0.5)\end{array}$ & $\begin{array}{r}3(0.8) \\
16(4.2) \\
2(1.6) \\
T \\
1(0.5) \\
23(5.6)\end{array}$ & $\begin{array}{l}4(0.6) \\
9(1.3) \\
3(0.7) \\
T \\
T \\
16(1.8)\end{array}$ & $\begin{array}{l}2(1.2) \\
4(0.5) \\
T 1 \\
T \\
1(0.6) \\
8(1.3)\end{array}$ & $\begin{array}{l}3(0.5) \\
10(0.7) \\
0 \\
\mathrm{~T} \\
\mathrm{~T} \\
13(1.4)\end{array}$ & $\begin{array}{l}4(0.6) \\
12(3.1) \\
0 \\
T \\
1(0.3) \\
17(3.1)\end{array}$ \\
\hline $\begin{array}{l}\text { Forbs } \\
\text { Balsamorhiza hookeri } \\
\text { Iva axillaris } \\
\text { Phlox hoodii } \\
\text { Phlox longifolia } \\
\text { Phoenicaulis } \\
\text { cheiranthoides } \\
\text { Sphaeralcea munroana } \\
\text { Other forbs } \\
\text { Unknown forbs } \\
\text { Total forbs }\end{array}$ & $\begin{array}{c}17(2.1) \\
0 \\
3(0.5) \\
17(3.9) \\
29(5.7) \\
T \\
6(2.0) \\
7(0.9) \\
79(1.2)\end{array}$ & $\begin{array}{c}1(0.9) \\
0 \\
1(0.3) \\
8(2.2) \\
3(1.3) \\
10(4.6) \\
0 \\
4(0.4) \\
27(6.3)\end{array}$ & $\begin{array}{l}2(0.4) \\
0 \\
0 \\
15(3.3) \\
2(0.9) \\
0 \\
0 \\
2(0.2) \\
21(4.6)\end{array}$ & $\begin{array}{r}4(0.8) \\
2(1.1) \\
2(0.6) \\
6(0.5) \\
10(3.6) \\
6(1.7) \\
11(1.4) \\
6(0.1) \\
47(5.2)\end{array}$ & $\begin{array}{r}18(1.9) \\
3(0.8) \\
2(1.8) \\
21(1.3) \\
\\
27(7.9) \\
0 \\
3(0.4) \\
6(0.8) \\
80(2.8)\end{array}$ & $\begin{array}{rr}3 & (0.8) \\
1 & (0.6) \\
1 & (0.3) \\
14 & (2.5) \\
& \\
9 & (4.3) \\
3 & (2.4) \\
T & \\
3 & (0.5) \\
35 & (1.3)\end{array}$ & $\begin{array}{l}1(0.6) \\
0 \\
7(4.3) \\
7(2.8) \\
T \\
0 \\
0 \\
6(1.4) \\
21(1.3)\end{array}$ \\
\hline $\begin{array}{l}\text { Shrubs } \\
\text { Artemisia arbuscula/A. } \\
\text { tridentata spp. } \\
\text { wyomingensis } \\
\text { Atriplex confertifolia } \\
\text { Other shrubs } \\
\text { Total shrubs }\end{array}$ & $\begin{array}{l}17(0.8) \\
0 \\
T \\
17(0.9)\end{array}$ & $\begin{array}{l}59(6.2) \\
\mathrm{T} \\
\mathrm{T} \\
60(6.0)\end{array}$ & $\begin{array}{l}56(1.7) \\
T \\
0 \\
56(1.6)\end{array}$ & $\begin{array}{l}37(6.1) \\
T \\
0 \\
37(6.2)\end{array}$ & $\begin{array}{l}12(3.3) \\
0 \\
T \\
12(3.4)\end{array}$ & $\begin{aligned} 52 & (1.8) \\
0 & \\
0 & \\
52 & (1.8)\end{aligned}$ & $\begin{array}{r}58(4.5) \\
4(0.3) \\
1(0.5) \\
63(4.2)\end{array}$ \\
\hline
\end{tabular}

ISpecies comprising at least $1 \%$ of any seasonal diet; complete species list found in McInnis (1985).

2Number of fecal groups comprising the mean of 3 samples per season.

Trace; less than $0.5 \%$. 


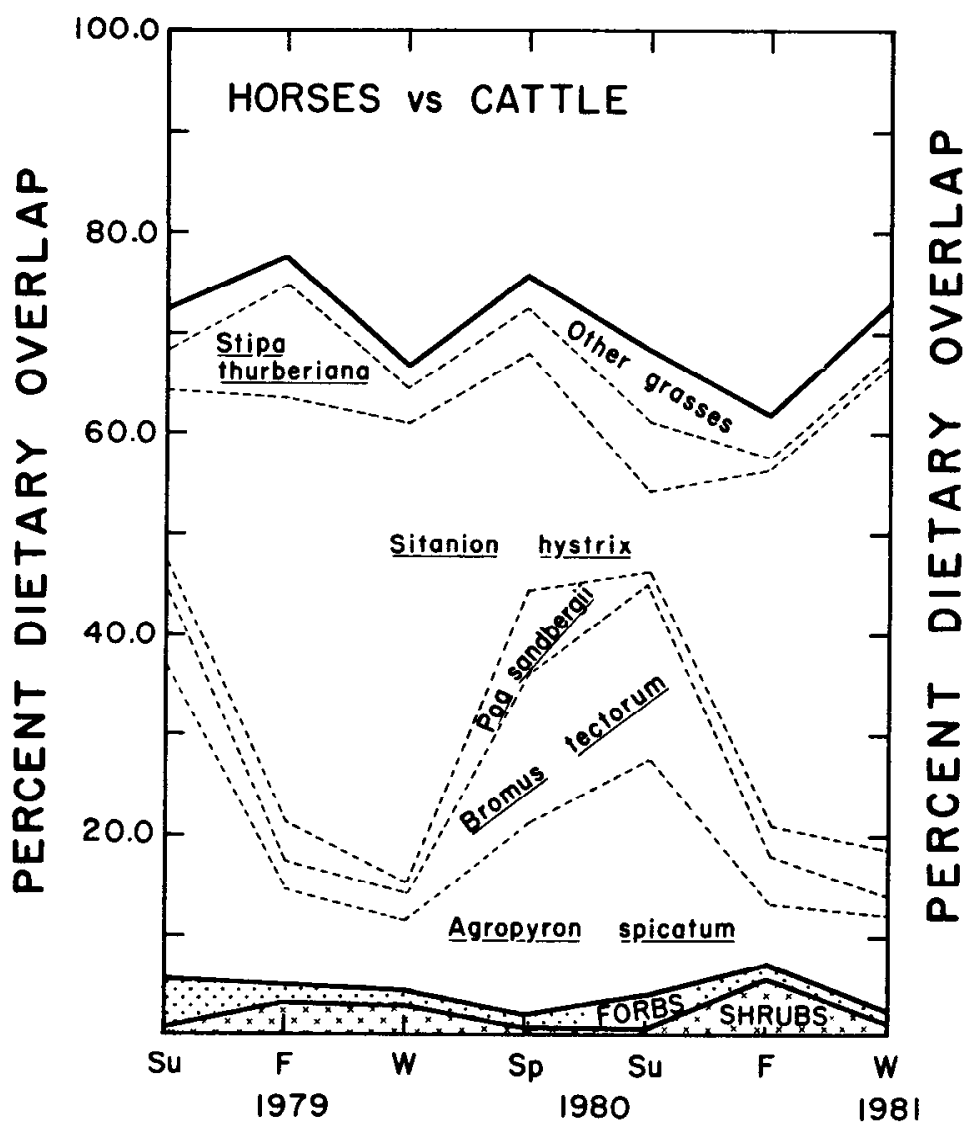

SEASONS

Fig. 1. Seasonal dietary overlap between feral horses and cattle. Topmost line represents total overlap.

Longleaf phlox (Phlox longifolia Nutt.), wallflower phoenicaulis (Phoenicaulis cheiranthoides Nutt.), and Hooker balsamroot (Balsamorhiza hookeri Nutt.) were the most heavily consumed forbs. During fall, forbs became less available due to advancing phenological development, and pronghorn switched to a diet dominated by shrubs. Low sagebrush and Wyoming big sagebrush could not be distinguished microhistologically, but collectively formed more than $50 \%$ of fall and winter diets.

\section{Dietary Overlap}

Of 21 taxa identified in the collective annual diets of feral horses and cattle, $81 \%$ were used in common. Dietary overlap averaged $70 \%$ on an annual basis, and exhibited modest but inconsistent seasonal variation (Fig. 1). When pooled across seasons, grasses formed $94 \%$ of total overlap. Collectively, bottlebrush squirreltail and bearded bluebunch wheatgrass formed roughly $50 \%$ or more of total overlap every season. Cheatgrass brome contributed nearly $30 \%$ of total overlap in summer 1980 , but only $10 \%$ in summer 1979. Horses and cattle consumed more than twice the amount of this grass during the summer of 1980 (Tables 1 and 2), possibly because greater mean precipitation during May-June 1980 (22.6 $\mathrm{mm}$ ) compared with the same period in $1979(11.7 \mathrm{~mm})$ may have increased the yield and retarded phenological development of this species resulting in increased palatability.

Twelve $(48 \%)$ of 25 forage species identified in the collective annual diets of feral horses and pronghorn were used commonly by both species of ungulates. On an annual basis, overlap averaged $16 \%$ and ranged from 7 to $26 \%$ (Fig. 2). Diets were most similar during winter months, and least similar in summer. When pooled across seasons and years, grasses (principally Sandberg bluegrass) contributed $70 \%$ of total overlap.

Thirty-one forage species were observed in the collective diets of cattle and pronghorn. Thirteen ( $42 \%)$ of these were consumed by
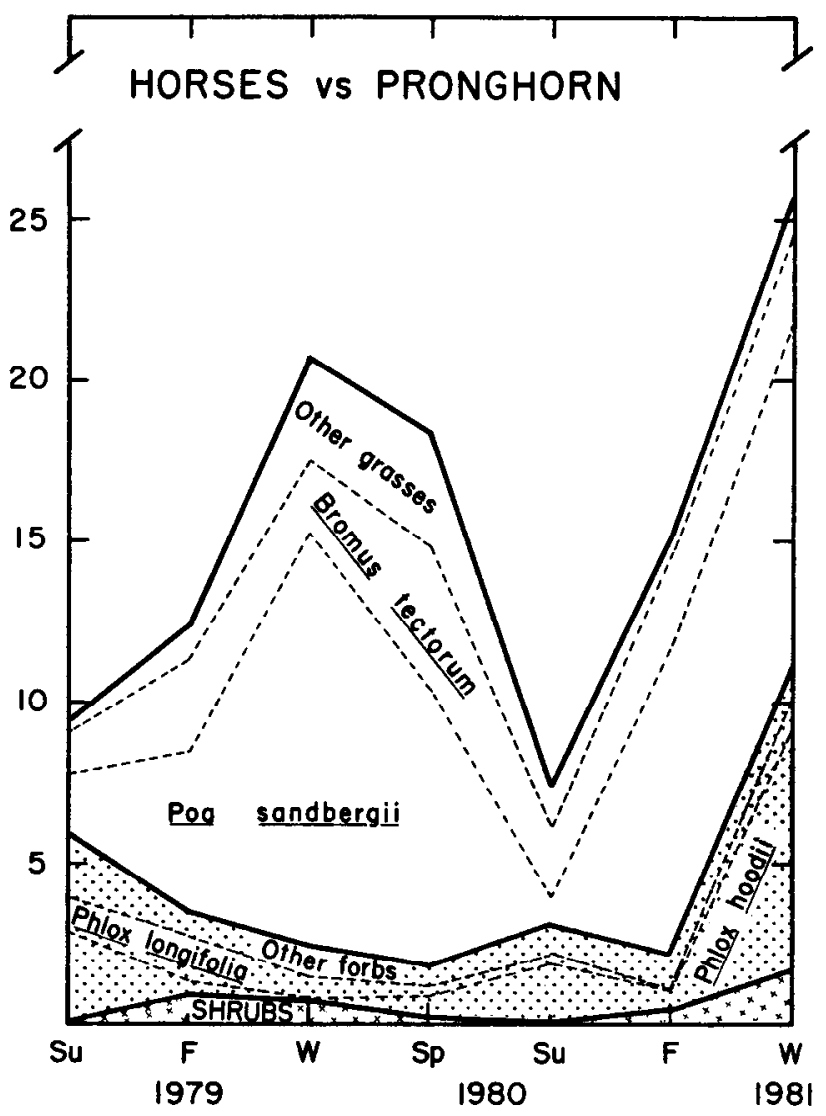

SEASONS

Fig. 2. Seasonal dietary overlap between feral horses and pronghorn. Topmost line represents total overlap.

both species of herbivores. As in the comparison of pronghorn with horses, dietary overlap between pronghorn and cattle was not high. Although the index clearly peaked during spring, trends in other seasonal fluctuations were inconsistent among years (Fig. 3). On an annual basis, the index averaged $14 \%$, and reached its lowest level during winter 1980. Common use of Sandberg bluegrass and cheatgrass brome collectively formed roughly $50 \%$ or more of the total overlap during most seasons.

\section{Forage Quality}

Seasonal levels of $\mathrm{CP}$ in important forages (Table 4) agreed with other studies conducted in the Great Basin (Hickman 1975, Spalinger 1980). Concentrations of CP in all forage classes were similar in spring, generally declined through summer and fall, and reached their lowest levels in winter. This trend was most pronounced in grasses and least in shrubs. Acid-detergent fiber content of all forage classes increased with decreasing levels of $\mathrm{CP}$ as the growing season progressed (Table 4). On the average, shrubs varied less than grasses or forbs in ADF throughout the year, and contained less ADF during all but spring months when levels were similar among all forage classes.

\section{Dietary Quality}

In most cases, estimates of dietary quality were based on forage species of which more than $95 \%$ were analyzed for CP and ADF. However, only about $75 \%$ of pronghorn diets in fall and winter were based on analyzed plants.

For each species of ungulate, dietary CP peaked in spring, steadily declined through the year, and reached its lowest level in winter (Table 5). Although all herbivores consumed diets containing roughly $16 \% \mathrm{CP}$ during spring, pronghorn selected plants containing consistently higher levels of $\mathrm{CP}$ than horses or cattle in 
Table 4. Seasonal mean composition (\% dry matter) of crude protein and acid-detergent fiber in fornges available to feral horses, cattle and pronghom.

\begin{tabular}{|c|c|c|c|c|c|}
\hline \multirow[t]{2}{*}{ Species/Season } & \multirow[b]{2}{*}{$\mathbf{n}$} & \multicolumn{2}{|c|}{ Crude Protein } & \multicolumn{2}{|c|}{$\begin{array}{c}\text { Acid-Detergen } \\
\text { Fiber }\end{array}$} \\
\hline & & $\overline{\boldsymbol{x}}$ & SE & $\bar{x}$ & SE \\
\hline \multicolumn{6}{|l|}{ Graminoids } \\
\hline \multicolumn{6}{|c|}{ Agropyron spicatum } \\
\hline spring & 3 & 16.3 & 2.9 & 34.2 & 2.5 \\
\hline summer & 3 & 5.7 & 1.9 & 47.4 & 2.2 \\
\hline fall & 2 & 3.7 & 0.6 & 54.3 & 1.6 \\
\hline winter & 4 & 2.3 & 0.3 & 57.1 & 1.2 \\
\hline \multicolumn{6}{|c|}{ Bromus tectorum } \\
\hline spring & 3 & 17.4 & 4.0 & 23.6 & 5.4 \\
\hline summer & 3 & 6.1 & 2.8 & 43.2 & 3.4 \\
\hline fall & 3 & 3.0 & 0.3 & 52.5 & 1.1 \\
\hline winter & 1 & 2.3 & & 54.9 & \\
\hline \multicolumn{6}{|c|}{ Poa sandbergii } \\
\hline spring & 3 & 13.9 & 3.6 & 31.0 & 4.0 \\
\hline summer & 3 & 3.0 & 0.3 & 49.0 & 0.6 \\
\hline fall & 3 & 4.3 & 0.8 & 51.1 & 1.3 \\
\hline winter & 2 & 6.8 & 0.0 & 51.2 & 0.4 \\
\hline \multicolumn{6}{|c|}{ Sitanion hystrix } \\
\hline spring & 3 & 17.5 & 3.1 & 34.9 & 3.1 \\
\hline summer & 2 & 6.5 & 3.3 & 48.2 & 3.7 \\
\hline fall & 3 & 6.1 & 1.5 & 56.9 & 0.9 \\
\hline winter & 4 & 3.7 & 0.1 & 54.7 & 1.3 \\
\hline \multicolumn{6}{|c|}{ Stipa thurberiana } \\
\hline spring & 3 & 14.0 & 1.7 & 38.7 & 6.6 \\
\hline summer & 2 & 6.5 & 1.6 & 50.4 & 3.6 \\
\hline fall & 2 & 4.0 & 1.3 & 51.6 & 0.6 \\
\hline winter & 2 & 3.3 & 0.4 & 56.9 & 2.3 \\
\hline \multicolumn{6}{|l|}{ Forbs } \\
\hline \multicolumn{6}{|c|}{ Phlox longifolia } \\
\hline spring & 1 & 14.2 & & 33.0 & \\
\hline summer & 2 & 7.2 & 1.5 & 47.1 & 0.6 \\
\hline winter & 2 & 5.8 & 0.1 & 62.3 & 1.1 \\
\hline \multicolumn{6}{|c|}{ Balsamorhiza hookeri } \\
\hline spring & 1 & 11.0 & & 25.6 & \\
\hline summer & 1 & 10.2 & & 32.6 & \\
\hline \multicolumn{6}{|c|}{ Phoenicaulis cheiranthoides } \\
\hline spring & 1 & 27.3 & & 19.0 & \\
\hline summer & 1 & 10.8 & & 30.3 & \\
\hline \multicolumn{6}{|l|}{ Iva axillaris } \\
\hline summer & 1 & 11.0 & & 27.3 & \\
\hline fall & 1 & 17.0 & & 30.5 & \\
\hline \multicolumn{6}{|l|}{ Shrubs } \\
\hline Artemisia & & & & & \\
\hline summer & 1 & 10.0 & & 32.6 & \\
\hline fall & 1 & 9.3 & & 34.8 & \\
\hline $\begin{array}{l}\text { Artemisia tr } \\
\text { wyomingen }\end{array}$ & & & & & \\
\hline spring & 1 & 14.5 & & 23.7 & \\
\hline summer & 1 & 13.9 & & 26.2 & \\
\hline fall & 3 & 11.3 & 0.3 & 30.0 & 2.0 \\
\hline winter & 3 & 10.5 & 0.6 & 32.1 & 0.5 \\
\hline Atriplex cor & & & & & \\
\hline spring & 1 & 15.6 & & 15.9 & \\
\hline summer & 1 & 14.6 & & 19.0 & \\
\hline fall & 2 & 8.4 & 0.0 & 25.9 & 5.4 \\
\hline winter & 3 & 8.2 & 0.8 & 30.8 & 1.2 \\
\hline Ceratoides & & & & & \\
\hline spring & 1 & 10.8 & & 46.3 & \\
\hline summer & 1 & 10.2 & & 42.2 & \\
\hline fall & 2 & 7.2 & 1.7 & 44.8 & 0.1 \\
\hline winter & 1 & 8.8 & & 41.3 & \\
\hline
\end{tabular}

every other season (Table 5). Within seasons, significant differences were not observed in dietary CP between horses and cattle. Levels of ADF in all ungulate diets were lowest in spring, increased steadily through the year, and generally reached their highest levels in winter. Pronghorn selected diets containing less ADF than horses or cattle during every season. Dietary ADF was signifi-
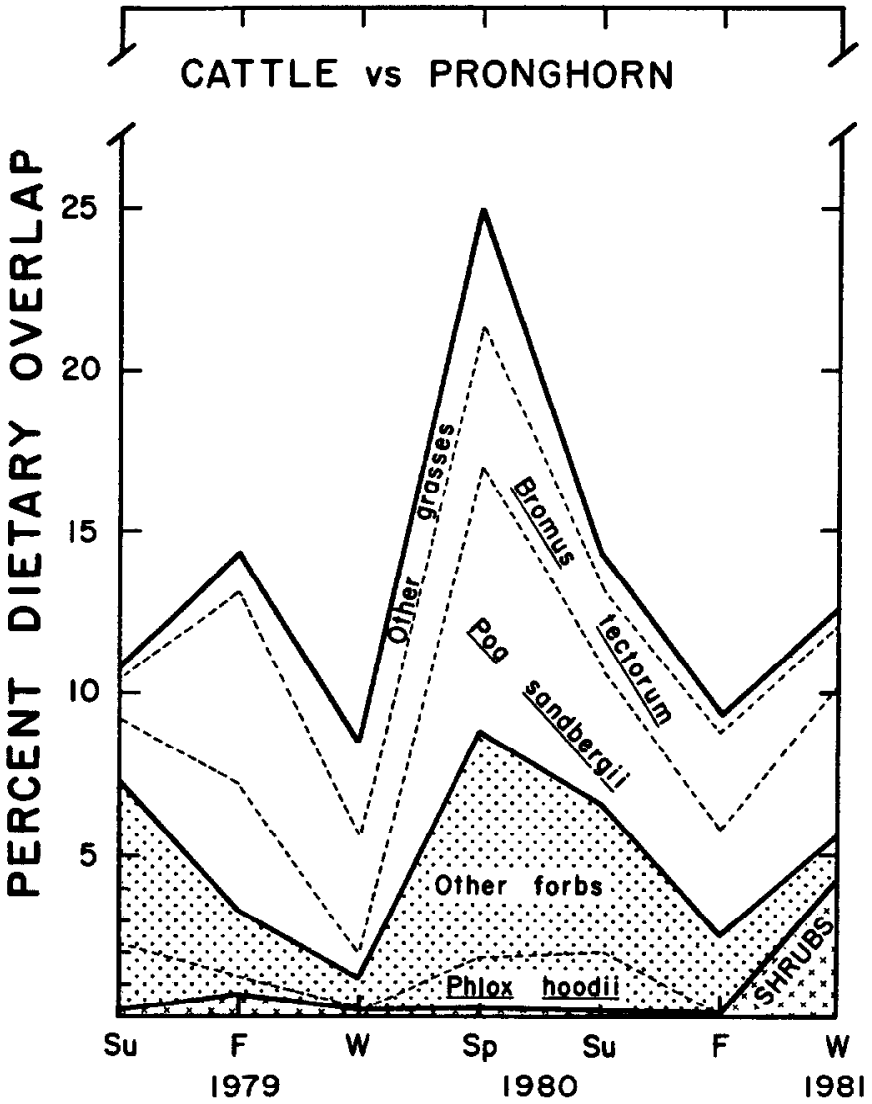

SEASONS

Fig. 3. Seasonal dietary overlap between cattle and pronghorn. Topmost line represents total overlap.

Table 5. Mean dietary composition ( $\%$ dry matter) of crude protein and acid-detergent fiber for feral horses, cattle, and pronghom.1

\begin{tabular}{|c|c|c|c|c|c|c|}
\hline \multirow[b]{2}{*}{ Season } & \multicolumn{2}{|c|}{ Feral Horses } & \multicolumn{2}{|c|}{ Cattle } & \multicolumn{2}{|c|}{ Pronghorn } \\
\hline & $\bar{x}$ & SE & $\bar{x}$ & $\overline{\mathrm{SE}}$ & $\overline{\bar{x}}$ & SE \\
\hline $\begin{array}{l}\text { Crude Protein } \\
\text { Summer } 1979\end{array}$ & $6.2^{*}$ & 0.1 & $6.1^{\circ}$ & 0.1 & $9.8^{\mathrm{a}}$ & 0.2 \\
\hline Fall 1979 & $5.2^{\mathrm{b}}$ & 0.1 & $5.5^{b}$ & 0.1 & $9.2^{\mathrm{b}}$ & 0.1 \\
\hline Winter 1980 & $4.3^{\mathrm{e}}$ & 0.2 & $\overline{4.2^{c}}$ & 0.3 & $8.3^{c}$ & 0.1 \\
\hline Spring 1980 & $\overline{15.8^{d}}$ & 0.1 & $\overline{16.5^{d}}$ & 0.2 & $\underline{16.0^{d}}$ & 0.5 \\
\hline Summer 1980 & $6.5^{n}$ & 0.1 & $5.8^{\mathrm{eb}}$ & 0.1 & $\overline{9.5^{\mathrm{ab}}}$ & 0.1 \\
\hline Fall 1980 & $5.4^{b}$ & 0.1 & $5.7^{\mathrm{ab}}$ & 0.1 & $8.7^{c}$ & 0.1 \\
\hline Winter 1981 & $4.2^{c}$ & 0.1 & $\overline{4.1^{\mathrm{c}}}$ & 0.03 & $8.5^{c}$ & 0.2 \\
\hline $\begin{array}{l}\text { Acid-Detergent } \\
\text { Summer } 1979\end{array}$ & $46.6^{\circ}$ & 01 & $46.5^{2}$ & 03 & $51^{\prime \prime}$ & \\
\hline Fall 1979 & $\overline{54.0^{\mathrm{bc}}}$ & 0.1 & $53.9^{\mathrm{b}}$ & 0.2 & $38.8^{b}$ & $\begin{array}{l}0.1 \\
0.8\end{array}$ \\
\hline $\begin{array}{l}\text { Winter } 1980 \\
\text { Spring } 1980\end{array}$ & $\begin{array}{l}\overline{54.6^{\mathrm{c}}} \\
32.9^{\mathrm{d}}\end{array}$ & $\begin{array}{l}0.2 \\
0.7\end{array}$ & $\begin{array}{l}\overline{51.9^{c}} \\
31.8^{\mathrm{d}}\end{array}$ & $\begin{array}{l}0.8 \\
0.2\end{array}$ & $\begin{array}{l}43.2^{\mathrm{c}} \\
25.6^{\mathrm{d}}\end{array}$ & $\begin{array}{l}0.2 \\
0.1\end{array}$ \\
\hline $\begin{array}{l}\text { Summer } 1980 \\
\text { Fall } 1980\end{array}$ & $\overline{\frac{46.8^{2}}{52.9^{4}}}$ & $\begin{array}{l}0.6 \\
0.2\end{array}$ & $\overline{\frac{46.5^{2}}{51.6^{c}}}$ & $\begin{array}{l}0.2 \\
0.4\end{array}$ & $\begin{array}{l}35.9^{a} \\
40.0^{b}\end{array}$ & $\begin{array}{l}0.6 \\
0.7\end{array}$ \\
\hline Winter 1981 & $\overline{55.2^{\circ}}$ & 0.3 & $\overline{52.5^{\circ}}$ & 0.3 & $41.5^{\circ}$ & 0.9 \\
\hline
\end{tabular}

'Ungulate and seasonal effects significant ( $P<0.01)$ within each nutritional category; $N=3$; means of each nutritional category compared within herbivore species across seasons are not significantly different $(P>0.05)$ if followed by the same superscript; means compared across herbivores within seasons are not significantly different $(P>0.05)$ if underlined. 
Table 6. Results of t test comparing pooled winter diets (1980 and 1981) of feral horses and cattle.'

\begin{tabular}{|c|c|c|c|c|}
\hline \multirow[b]{2}{*}{ Species } & \multicolumn{2}{|c|}{$\begin{array}{l}\text { Mean Dietary } \\
\text { Composition }\end{array}$} & \multirow{2}{*}{$\begin{array}{c}\text { Computed } \\
\mathrm{t} \text { values }\end{array}$} & \multirow[b]{2}{*}{ Significance ${ }^{2}$} \\
\hline & Horses & Cattle & & \\
\hline $\begin{array}{l}\text { Agropyron spicatum } \\
\text { Bromus tectorum } \\
\text { Poa sandbergii } \\
\text { Sitanion hysirix } \\
\text { Stipa thurberiana }\end{array}$ & $\begin{array}{r}9 \\
3 \\
12 \\
47 \\
11\end{array}$ & $\begin{array}{r}11 \\
5 \\
3 \\
60 \\
2\end{array}$ & $\begin{array}{r}-0.6000 \\
-1.1780 \\
2.0467 \\
-2.7646 \\
5.6554\end{array}$ & $\begin{array}{l}\text { ns } \\
\text { ns } \\
* \\
* * \\
* * *\end{array}$ \\
\hline $\begin{array}{l}\text { Total graminoids } \\
\text { Total forbs } \\
\text { Total shrubs }\end{array}$ & $\begin{array}{r}88 \\
10 \\
3\end{array}$ & $\begin{array}{r}86 \\
1 \\
14\end{array}$ & $\begin{array}{r}0.8997 \\
5.1512 \\
-3.7163\end{array}$ & $\begin{array}{c}\text { ns } \\
+* * \\
* *\end{array}$ \\
\hline
\end{tabular}

Based on paired $t$ test comparing monthly proportions of each food item between horses and cattle; $n=6$.

${ }^{2} \mathrm{~ns}, *, * *$, and ${ }^{* * *}$ respectively refer to not significant; $P<0.1 ; P<0.05$; and $P<0.01$.

cantly different between horses and cattle only during winter.

\section{Discussion and Conclusions}

While forbs and shrubs may be locally important in diets of free-ranging horses (Hansen 1976, Krysl et al. 1984) and cattle (Uresk and Rickard 1976, Johnson 1979), most studies concluded these ungulates are primarily grass consumers throughout the year (Skiles 1984). Within shrub-steppe communities horses and cattle consumed year-round diets containing roughly $90 \%$ grass (Vavra and Sneva 1978). Most workers found substantial dietary overlap between these herbivores in a variety of vegetation types (Olsen and Hansen 1977, Salter and Hudson 1979, Hanley and Hanley 1982, Krysl et al. 1984). Dietary overlap between feral horses and cattle grazing common sagebrush-grassland range in eastern Oregon average 67,69 , and $72 \%$ during spring, summer, and winter, respectively (Vavra and Sneva 1978). Conversely, pronghorn sparsely use grasses (Skiles 1984). Previous comparisons have shown little overlap in food habits of sympatric pronghorn and horses (Olsen and Hansen 1977, Meeker 1979, and Hanley and Hanley 1982), or pronghorn and cattle (Olsen and Hansen 1977, Johnson 1979, Hanley and Hanley 1982).

In this study, ungulates were classified as principally grass consumers (horses and cattle), or forb and shrub consumers (pronghorn). Horses and cattle showed predilection for many of the same forages, and dietary overlap was substantial (62-78\%) every season. Conversely, pronghorn shared relatively few food items with horses or cattle. These results support our first hypothesis and agree with Hanley's (1982) food selection framework.

Dietary overlap is not sufficient evidence for exploitative competition (Colwell and Futuyma 1971), and consequences of overlap partially depend upon availability of the resource. In one case exploitation would not occur between 2 species sharing a common abundant resource which is not limiting (Sale 1974, Lawlor 1980). On the other hand, competition may increase if availability is reduced by common utilization (Nelson 1982). Interspecific competition must be judged on the basis of 2 criteria (Wagner 1983): (1) 2 species compete when they share a resource that is present in short supply, and (2) in using the resource, each species reduces the other's population performance to levels below what these measures would be in the absence of the other species. Although it is beyond the scope of this study to test the hypothesis of competition among horses, cattle, and pronghorn, our data provide insight into potential exploitative interactions among these species.

The theory of competitive exclusion (Hardin 1960) proposed 2 species cannot coexist on the same limiting resource. Ecologically similar species may coexist by partitioning resources among themselves (Schoener 1974), particularly during the lean season (Smith et al. 1978). In the present study, winter was the presumed season of lowest forage availability. Horses and cattle consumed signifi- cantly different proportions of some food items during this season (Table 6), but total overlap still averaged 70\%(Fig. 1). High dietary overlap and similarity of habitat selection (McInnis 1985) suggest competition could occur between horses and cattle.

In the pronghorn-horse and pronghorn-cattle comparisons, low dietary overlap (Fig. 2 and 3) may suggest a wider buffer between noncompetitive coexistence and exploitative competition. However, Vavra and Sneva (1978) demonstrated a potential for higher levels of dietary similarity (20-49\%) when drought reduced availability of foods preferred by pronghorn and they switched to diets high in large perennial grasses. Wagner (1983) concluded competition for forage between grazers and browsers was most likely to occur on depleted range where food habits of such herbivores converged.

Our second hypothesis stated pronghorn would select diets of higher nutritional quality than horses or cattle because of their smaller size and higher metabolic rate (Hanley and Hanley 1982). Indeed, pronghorn consumed consistently lower levels of ADF; and higher proportions of $\mathrm{CP}$ during every season except spring (Table 5). The effect of higher metabolic rates of small animals is that they require more protein and energy per unit weight than larger animals (Bell 1971, Schwartz and Ellis 1981). Schwartz et al. (1977) observed consumption of less fibrous diets by pronghorn than cattle, bison or sheep. This trend may reflect differences in feeding strategies of these herbivores. Large body size and large gastro-intestinal volume (e.g., horses and cattle) are adaptations to exploitation of high cellulose diets (grasses) while small body size and small gastro-intestinal volume (e.g., pronghorn) are adaptations to exploitation of high cell soluble diets (forbs and shrubs) (Hanley and Hanley 1982).

An essential difference between ruminant and cecal digestors is that in the former group, passage rate of digesta and intake is depressed by increasing levels of dietary fiber (Janis 1976, Hanley 1982). To maintain an adequate level of nutrient absorption, ruminants increase mass flow of digesta through the gastro-intestinal tract by selecting diets lower in fiber, especially when forage quality is limiting (Bell 1971, Janis 1976, Hanley 1982). In our study nutrient quality of most forages was lowest in winter (Table 4), and cattle selected diets containing significantly less ADF than horses (Table 5). We observed no other significant differences in the quality of diets consumed by horses and cattle.

Comparative data on the nutrition of free-ranging ungulates are scarce. Requirements of CP for domestic horses vary from $8.5 \%$ for maintenance to $14 \%$ during the first 3 months of lactation (NRC 1978). In the present study, newborn foals were observed between late March and May (McInnis 1985), and CP varied from roughly $4 \%$ in winter to nearly $16 \%$ in spring (Table 5). Based on comparison with percent $\mathrm{CP}$ requirements, horses in our study were apparently deficient during all but spring months. However, equids have evolved a digestive strategy which allows them to compensate for low quality forage by increasing throughput and intake rates (Janis 1976). Thus, horses may have received adequate levels of CP by processing more feed than ruminants.

Beef cows require $7-8 \% \mathrm{CP}$ for maintenance and $9-10 \%$ for lactation (NRC 1984). Raleigh (1970) reported cattle grazing sagebrush-bunchgrass vegetation in southeastern Oregon consumed diets containing about 19\% CP in early May and 3\% in mid-September. Cattle in the present study exceeded the requirements for lactation in early spring when calves were born, but dietary CP declined rapidly through summer and apparently failed to meet lactation requirements then (Table 5). Further declines through fall and winter caused diets to be deficient in CP during those periods.

Although nutritional requirements of pronghorn are largely unknown, Schwartz et al. (1977) speculated the CP requirement for deer (Ocodoileus spp.) may be comparable (6-7\% for maintenance and 13-16\% for growth). Smith and Malechek (1974) determined dietary CP of pronghorn declined from $22-10 \%$ between early May and late September on desert ranges in Utah, but 
concluded maintenance requirements were met throughout all periods studied. If pronghorn requirements for $\mathrm{CP}$ are similar to those of deer, diets consumed by pronghorn in our study were probably adequate for maintenance each season (Table 5). However, levels necessary for growth were reached only during spring. As forage matured during summer months, dietary CP declined, and may have been inadequate to meet the needs of lactating females.

Our estimates of dietary protein may have underestimated actual proportions consumed for 2 reasons: Firstly, hand-compounded samples contain less protein and more fiber than diets actually selected by free-ranging herbivores (Edlefsen et al. 1960, Kiesling et al. 1969). This may be especially true for pronghorn because their small mouths allow them to be more selective of plant parts than animals with large mouths (Hanley 1982). Secondly, estimates of dietary quality were based on forage species occasionally totaling less than actual amounts consumed. Since estimates for horses and cattle approached or reached $100 \%$ of actual diets, dietary CP and ADF of these herbivores was probably not markedly affected. However, CP and ADF levels in pronghorn diets during fall and winter were probably somewhat higher than suggested by our results (Table 5). Despite these deficiencies, our results provide an initial comparison of seasonal dietary quality for feral horses, cattle, and pronghorn.

\section{Literature Cited}

Anthony, R.G., and N.S. Smith. 1977. Ecological relationships between mule deer and white-tailed deer in southeastern Arizona. Ecol. Monog. 47:255-277.

Bell, R.H.V.1971. A grazing system in the Serengeti. Sci. Amer. 225:86-93.

Colwell, R.K., and D.J. Futuyma. 1971. On the measurements of niche breadth and overlap. Ecol. 52:567-576.

Demment, M.W., and P.J. Van Soest. 1985. A nutritional explanation for body-size patterns of ruminant and nonruminant herbivores. Amer. Natur. 125:641-672.

Eberhardt, L.L., A.K. Majorowicz, and J.A. Wilcox. 1982. Apparent rates of increase for 2 feral horse herds. J. Wildl. Manage. 46:367-374.

Edlefsen, J.L., C.W. Cook, and J.T. Blake. 1960. Nutrient content of the diet as determined by hand plucked and esophageal fistula samples. J. Anim. Sci. 19:560-567.

Franklin, J.F., and C.T. Dyrness. 1973. Natural vegetation of Oregon and Washington. USDA Forest Serv. Gen. Tech. Rep. PNW-8.

Hanley, T.A. 1982. The nutritional basis for food selection by ungulates. J. Range Manage., 35:146-151.

Hanley, T.A., and K.A. Hanley. 1982. Food resource partitioning by sympatric ungulates on Great Basin rangelands. J. Range Manage. 35:152-158.

Hansen, R.M. 1976. Foods of free-roaming horses in southern New Mexico. J. Range Manage. 29:347.

Hardin, G. 1960. The competitive exclusion principle. Science 131:1292-1297.

Hickman, 0.E. 1975. Seasonal trends in the nutritive content of important range forage species near Silver Lake, Oregon. USDA Forest Serv. Res. Paper PNW-187.

Hudson, R.J. 1976. Resource division within a community of large herbivores, Naturaliste Can. 103:153-167.

Hurlbert, S.H. 1978. The measurement of niche overlap and some relatives. Ecology 59:67-77.

Janis, C. 1976. The evolutionary strategy of the Equidae and the origins of rumen and cecal digestion. Evolution. 30:757-774.

Johnson, M.K. 1979. Foods of primary consumers on cold desert shrubsteppe of southcentral Idaho. J. Range Manage. 32:365-368.

Kiesling, H.E., A.B. Nelson, and C.H. Herbel. 1969. Chemical composition of tobosa grass collected by hand-plucking and esophageal-fistulated steers. J. Range Manage. 22:155-159.

Krysl, L.J., M.E. Hubbert, B.F. Sowell, G.D. Plumb, T.K. Jewett, M.A. Smith, and J.W. Wagsoner. 1984. Horses and cattle grazing in the Wyoming Red Desert, I. Food habits and dietary overlap. J. Range Manage. 37:72-76.
Lawlor, L.R. 1980. Overlap, similarity, and competition coefficients. Ecology 61:245-251.

McGuire, J.R. 1973. Wild free-roaming horses and burros within the natural system under the multiple-use concept. USDA Forest Serv. Environ. Statement. Washington, D.C.

McInnis, M.L. 1985. Ecological relationships among feral horses, cattle, and pronghorn in southeastern Oregon. Ph.D. Diss., Oregon State Univ., Corvallis.

McInnis, M.L., M. Vavra, and W.C. Krueger. 1983. A comparison of 4 methods used to determine the diets of large herbivores. J. Range Manage., 36:302-306.

Meeker, J.O. 1979. Interactions between pronghorn antelope and feral horses in northwestern Nevada. M.S. Thesis. Univ. Nevada, Reno.

Nebon, J.R. 1982. Relationships of elk and other large herbivores, p. 415-441. In: J.W. Thomas and D.E. Toweill (eds.), Elk of North America: ecology and management. Stackpole Books Harrisburg, Penn.

NRC. 1978. Nutrient requirements of domestic animals. No. 6. Nutrient requirements of horses. Nat. Acad. Sci., Nat. Res. Counc., Washington, D.C.

NRC. 1984. Nutrient requirements of domestic animals. No. 4. Nutrient requirements of beef cattle. Nat. Acad. Sci., Nat. Res. Counc., Washington, D.C.

Olsen, F.W., and R.M. Hansen. 1977. Food relations of wild free-roaming horses to livestock and big game, Red Desert, Wyoming. J. Range Manage. 30:17-20.

Raleigh, R.J.1970. Symposium on pasture methods for maximum production in beef cattle: Manipulation of both livestock and forage management to give optimum production. J. Anim. Sci. 30:108-114.

Sale, P.F. 1974. Overlap in resource use, and interspecific competition. Oecologia 17:245-256.

Salter, R.E., and R.J. Hudson. 1979. Feeding ecology of feral horses in western Alberta. J. Range Manage. 32:221-225.

Schoener, T.W. 1974. Resource partitioning in ecological communities. Science 185:27-39.

Schwartz, C.C., and J.E. Ellis, 1981. Feeding ecology and niche separation in some native and domestic ungulates on the shortgrass prairie. J. Appl. Ecol. 18:343-353.

Schwartz, C.C., J.G. Nagy, and R.W. Rice. 1977. Pronghorn dietary quality relative to forage availability and other ruminants in Colorado. $J$. Wildl. Manage. 41:161-168.

Skiles, J.W. 1984. A review of animal preference, p. 153-213. In: Nat. Resour. Counc. Nat. Acad. Sci., Developing strategies for rangeland management. Westview Press. Boulder, Colo.

Smith, A.D., and J.C. Malechek. 1974. Nutritional quality of summer diets of pronghorn antelope in Utah. J. Wildl. Manage. 38:792-798.

Smith, J.N.M., P.R. Grant, I.J. Abbott, and L.K. Abbott. 1978. Seasonal variation in feeding habits of Darwin's ground finches. Ecol. 59:1137-1150

Spalinger, D.D. 1980. Mule deer habitat evaluation based upon nutritional modeling. M.S. Thesis. Univ. Nevada, Reno.

Sparks, D.R., and J.C. Malechek. 1968. Estimating percentage dry weight in diets using a microscope technique. J. Range Mange. 21:264-265.

Steel, R.G., and J.H. Torrie. 1960. Principles and procedures of statistics. McGraw-Hill Book Co., Inc. New York.

Sullivan, J.T. 1962. Evaluation of forage crops by chemical analysis: a critique. Agron. J., 54:511-515.

Uresk, D.W., and W.H. Ricknrd. 1976. Diets of steers on a shrub-steppe rangeland in south-central Washington. J. Range Manage. 29:464-466.

Van Soest, P.J. 1963. Use of detergents in the analysis of fibrous feeds. II. A rapid method for the determination of fiber and lignin. J. Ass. Off. Anal. Chem., 46:829-835.

Van Soest, P.J., and R.H. Wine. 1967. Use of detergents in the analysis of fibrous feeds. IV. Determination of plant cell-wall constituents. J. Ass. Off. Anal. Chem. 50:50-55.

Vavra, M., and F.A. Sneva. 1978. Seasonal diets of 5 ungulates grazing in the cold desert biome, p. 435-437. In: D.N. Hyder (ed.), Proc. First Internat. Rangeland Cong., Soc. Range Manage.

Wagner, F.H. 1983. Status of wild horse and burro management on public rangelands. Trans. N. Amer. Wildl. \& Nat. Resour. Conf. 48:116-133.

Westoby, M. 1974. An analysis of diet selection by large generalist herbivores. Amer. Natur. 108:290-304.

Whittaker, R.H. 1975. Communities and ecosystems. MacMillan Publ., Co., New York 